\title{
The operator approach to the truncated multidimensional moment problem
}

https://doi.org/10.1515/conop-2019-0001

Received May 14, 2018; accepted January 2, 2019

\begin{abstract}
We study the truncated multidimensional moment problem with a general type of truncations. The operator approach to the moment problem is presented. The case where the associated operators form a commuting self-adjoint tuple is characterized in terms of the given moments. The case of the dimensional stability is characterized in terms of the prescribed moments as well. Some sufficient conditions for the solvability of the moment problem are presented. A construction of the corresponding solution is described by algorithms. Numerical examples of the construction are provided.
\end{abstract}

Keywords: moment problem, symmetric operator, extension

\section{Introduction}

Let us introduce some notations. As usual, we denote by $\mathbb{R}, \mathbb{C}, \mathbb{N}, \mathbb{Z}, \mathbb{Z}_{+}$the sets of real numbers, complex numbers, positive integers, integers and non-negative integers, respectively. By $\mathbb{Z}_{+}^{n}$ we mean $\mathbb{Z}_{+} \times \ldots \times \mathbb{Z}_{+}$, and $\mathbb{R}^{n}=\mathbb{R} \times \ldots \times \mathbb{R}$, where the Cartesian products are taken with $n$ copies. Let $\mathbf{k}=\left(k_{1}, \ldots, k_{n}\right) \in \mathbb{Z}_{+}^{n}$, $\mathbf{t}=\left(t_{1}, \ldots, t_{n}\right) \in \mathbb{R}^{n}$. We denote by $\mathbf{t}^{\mathbf{k}}$ the monomial $t_{1}^{k_{1}} t_{2}^{k_{2}} \ldots t_{n}^{k_{n}}$, and we let $|\mathbf{k}|=k_{1}+\ldots+k_{n}$. We also denote by $\mathfrak{B}\left(\mathbb{R}^{n}\right)$ the set of all Borel subsets of $\mathbb{R}^{n}$.

Let $\mathcal{K}$ be an arbitrary finite subset of $\mathbb{Z}_{+}^{n}$, and $\mathcal{S}=\left(s_{\mathbf{k}}\right)_{\mathbf{k} \in \mathcal{K}}$ an arbitrary set of real numbers. The truncated multidimensional moment problem consists of finding a (non-negative) measure $\mu$ on $\mathfrak{B}\left(\mathbb{R}^{n}\right)$ such that

$$
\int \mathbf{t}^{\mathbf{k}} d \mu(\mathbf{t})=s_{\mathbf{k}}, \quad \forall \mathbf{k} \in \mathcal{K} .
$$

The multidimensional moment problem (both the full and the truncated versions) turned out to be much more complicated than its one-dimensional prototype [1], [2], [13]. An operator-theoretical interpretation of the (full) multidimensional moment problem was given by Fuglede in [7]. It should be noticed that the operator approach to moment problems was introduced by Naimark in 1940-1943 and then developed by many authors, see historical notes in [28]. Elegant conditions for the solvability of the multidimensional moment problem in the case of the support on semi-algebraic sets were given by Schmüdgen in [17], [18]. Another conditions for the solvability of the multidimensional moment problem, using an extension of the moment sequence, were given by Putinar and Vasilescu, see [16], [21]. Developing the idea of Putinar and Vasilescu, we presented different conditions for the solvability of the two-dimensional moment problem and proposed an algorithm (which essentially consists of solving of linear equations) for a construction of the solutions set [25]. An analytic parametrization for all solutions of the two-dimensional moment problem in a strip was given in [27]. Another approach to multidimensional and complex moment problems (including truncated problems), using extension arguments for *-semigroups, has been developed by Cichoń, Stochel and Szafraniec,

\footnotetext{
^Corresponding Author: Sergey M. Zagorodnyuk: V. N. Karazin Kharkiv National University, School of Mathematics and Computer Sciences, Department of Higher Mathematics and Informatics, Svobody Square 4, 61022, Kharkiv, Ukraine, E-mail: Sergey.M.Zagorodnyuk@gmail.com; zagorodnyuk@karazin.ua
} 
see [3] and references therein. Still another approach for the two-dimensional moment problem was proposed by Ovcharenko in [14], [15].

In this paper we shall be focused on the truncated multidimensional moment problem. A general approach for this moment problem was given by Curto and Fialkow in their books [4] and [5]. These books entailed a series of papers by a group of mathematicians, see recent papers [6], [22], [24] and references therein. This approach includes an extension of the matrix of prescribed moments with the same rank. Effective optimization algorithms for the multidimensional moment problems were given in the book of Lasserre [11]. Another approach for truncated moment problems, using a notion of an idempotent, was presented by Vasilescu in [23]. Atomic solutions to various matrix truncated $K$-moment problems were studied by Kimsey and Woerdeman in [9]. There exists a connection of the truncated multidimensional moment problems with the completion problems for subnormal operators, see, e.g., [10]. Observe that the complexification of the real truncated moment problem needs the even dimension $d$. The complexification of the truncated multidimensional moment problem and the use of hyponormal operators was investigated by Kimsey and Putinar in [8]. We should also mention recent papers [29], [19] on the subject.

Even in the one-dimensional case $(n=1)$ the operator approach is not effective for all types of truncations $\mathcal{K}$. Thus, we need to consider some admissible types of truncations, where one can get solutions. We shall deal with truncations of the form $\mathcal{K}=K+K$, where $K$ is admissible (see Definition 1 below). This type of admissible subsets appeared in the work of Kimsey and Woerdeman [9]. It also appeared implicitly in the paper of Laurent and Mourrain [12] (see the definition of a connected set on page 89 therein). Laurent and Mourrain investigated the case of flatness for the corresponding moment problem. Kimsey and Woerdeman mainly worked with another type of truncations $\mathcal{K}(\neq K+K)$, and constructed atomic solutions under some conditions.

The second feature of the truncated case is that we need to take care that the associated multiplication operators in the associated Hilbert space are well-defined. In Theorem 1 we give necessary conditions for the solvability of the moment problem which guarantee that the associated operators are well-defined. While condition (8) is well known, we did not meet condition (17) in this general context. For the case of the dimensional stability, see, e.g. [22, Lemma 2.3].

If all the above is done, we come to a problem of an extension of commuting symmetric operators to a commuting self-adjoint tuple, see Proposition 1. Of course, one especially needs explicit conditions, under which the extensions exist and one can construct a solution of the moment problem. It should be noticed that, besides some special cases of small size truncations, the notion of flatness turned out to be the most useful and explicit. Its numerical applications were discussed in the book of Lasserre [11] (see, in particular, Algorithm 4.1. on page 78).

In paper [22] Vasilescu introduced a notion of the dimensional stability, which used the geometry of the associated Hilbert spaces. He showed that the dimensional stability is equivalent to flatness (in the case of truncations described in Example 1, part 1 below). We provide a definition of the dimensional stability in our case (Definition 3) and present explicit numerical conditions which characterize this notion (Theorem 5).

We introduce a definition of the completely self-adjoint case (Definition 2) and provide explicit numerical conditions which characterize this notion (Theorem 4) as well. We briefly consider the interrelation between the dimensional stability and the complete self-adjointness (Theorem 6).

The main result of the paper is Theorem 7 which provides explicit numerical conditions which ensure that the moment problem (1) is solvable. These conditions do not reduce to the case of the dimensional stability, since the associated operators are allowed to be nonself-adjoint. The construction of the corresponding solution of the moment problem is described in details by Algorithms 1,2. Numerical examples, which illustrate the above results, are also given.

Notations. Besides the notations given above we shall use the following conventions. By $\mathbb{Z}_{k, l}$ we mean all integers $j$ satisfying the following inequality: $k \leq j \leq l ;(k, l \in \mathbb{Z})$. For a complex number $z$, we write $\operatorname{Re} z$ for its real part and $\operatorname{Im} z$ for its imaginary part. If $H$ is a Hilbert space then $(\cdot, \cdot)_{H}$ and $\|\cdot\|_{H}$ denote the scalar product and the norm in $H$, respectively. Indices may be omitted in obvious cases. For a linear operator $A$ in $H$, we denote by $D(A)$ its domain, by $R(A)$ its range, and by $A^{*}$ its adjoint operator if it exists. We denote the inverse of $A$ by $A^{-1}$ if $A$ is invertible, and the closure of $A$ by $\bar{A}$ if $A$ is closable. If $A$ is bounded then $\|A\|$ denotes its 
norm. For a set $M \subseteq H$ we denote by $\bar{M}$ the closure of $M$ in the norm of $H$. By Lin $M$ we mean the set of all linear combinations of elements from $M$, and $\operatorname{span} M:=\overline{\operatorname{Lin} M}$. By $E_{H}$ we denote the identity operator in $H$, i.e. $E_{H} \chi=x, \chi \in H$. In obvious cases we may omit the index $H$. If $H_{1}$ is a subspace of $H$, then $P_{H_{1}}=P_{H_{1}}^{H}$ denotes the orthogonal projection of $H$ onto $H_{1}$.

\section{Necessary conditions for the solvability of the moment problem}

Consider the following operator $W_{j}$ on $\mathbf{Z}_{+}^{n}$ :

$$
W_{j}\left(k_{1}, \ldots, k_{j-1}, k_{j}, k_{j+1}, \ldots, k_{n}\right)=\left(k_{1}, \ldots, k_{j-1}, k_{j}+1, k_{j+1}, \ldots, k_{n}\right),
$$

for $j=1, \ldots, n$. Thus, the operator $W_{j}$ increases the $j$-th coordinate.

Definition 1. A finite subset $K \subset \mathbb{Z}_{+}^{n}$ is said to be admissible, if the following conditions hold:

1) $\mathbf{0}=(0, \ldots, 0) \in K$;

2) $\forall \mathbf{k} \in K \backslash\{\mathbf{0}\}$,

$$
\mathbf{k}=W_{a_{|\mathbf{k}|}} W_{a_{|\mathbf{k}|-1}} \ldots W_{a_{1}} \mathbf{0}
$$

for some $a_{j} \in\{1, \ldots, n\}$, and

$$
\tilde{\mathbf{k}}_{r}:=W_{a_{r}} \ldots W_{a_{1}} \mathbf{0} \in K, \quad \forall r=1,2, \ldots,|\mathbf{k}| .
$$

We provide below some important examples of admissible sets.

Example 1. 1) Let $K=K_{r}=\left\{\mathbf{k} \in \mathbb{Z}_{+}^{n}:|\mathbf{k}| \leq r\right\}, r \in \mathbb{Z}_{+}$. Then $K$ is admissible, since $\forall \mathbf{k}=\left(k_{1}, \ldots, k_{n}\right) \in K \backslash\{\mathbf{0}\}$,

$$
\mathbf{k}=W_{n}^{k_{n}} W_{n-1}^{k_{n-1}} \ldots W_{1}^{k_{1}} \mathbf{0}
$$

2) For $d_{1}, \ldots, d_{n} \in \mathbb{Z}_{+}$, let

$$
K=K_{d_{1}, d_{2}, \ldots, d_{n}}:=\left\{\mathbf{k}=\left(k_{1}, \ldots, k_{n}\right) \in \mathbb{Z}_{+}^{n}: k_{1} \leq d_{1}, k_{2} \leq d_{2}, \ldots, k_{n} \leq d_{n}\right\} .
$$

The set $K_{d_{1}, d_{2}, \ldots, d_{n}}$ is admissible, since $\forall \mathbf{k}=\left(k_{1}, \ldots, k_{n}\right) \in K \backslash\{\mathbf{0}\}$,

$$
\mathbf{k}=W_{n}^{k_{n}} W_{n-1}^{k_{n-1}} \ldots W_{1}^{k_{1}} \mathbf{0}
$$

Notice that the truncated two-dimensional moment problem with rectangular data appeared in [8], [29]. The general case of the set $K_{d_{1}, d_{2}, \ldots, d_{n}}$ was proposed to the author by Vasilescu (private communication).

Suppose that for an admissible finite set $K \subset \mathbb{Z}_{+}^{n}$ the moment problem (1), with

$$
\mathcal{K}=K+K:=\left\{\mathbf{k} \in \mathbb{Z}_{+}^{n}: \mathbf{k}=\mathbf{u}+\mathbf{v}, \mathbf{u}, \mathbf{v} \in K\right\},
$$

and some $\mathcal{S}=\left(s_{\mathbf{k}}\right)_{\mathbf{k} \in \mathcal{K}}$, has a solution $\mu$. Let us investigate which properties of the data $\mathcal{S}$ this fact yields.

The first property is the usual positivity condition. For practical purposes, we will assume below that the elements of $K$ are indexed by a single index i.e., we assume

$$
K=\left\{\mathbf{k}_{0}, \mathbf{k}_{1}, \ldots, \mathbf{k}_{\rho}\right\}
$$

with $\rho+1=|K|$. Consider an arbitrary polynomial of the following form:

$$
p(\mathbf{t})=\sum_{j=0}^{\rho} \alpha_{j} \mathbf{t}^{\mathbf{k}_{j}}, \quad \alpha_{j} \in \mathbb{C} .
$$

Evaluating $\int|p|^{2} d \mu$, we get

$$
\Gamma \geq 0
$$


where

$$
\Gamma:=\left(s_{\mathbf{k}_{j}+\mathbf{k}_{m}}\right)_{j, m=0}^{\rho} .
$$

We now suppose that for an admissible finite set $K \subset \mathbb{Z}_{+}^{n}$ the moment problem (1), with $\mathcal{K}=K+K$ and some $\mathcal{S}=\left(s_{\mathbf{k}}\right)_{\mathbf{k} \in \mathcal{K}}$, is given and condition (8) holds (we do not require that the moment problem is solvable). Let $\mathfrak{L}$ denote the set of polynomials of the form (7). Observe that $\mathfrak{L}$ forms a vector space. Consider the following functional on $\mathfrak{L}$ :

$$
\langle p, q\rangle=\sum_{j, m=0}^{\rho} \alpha_{j} \overline{\beta_{m}} S_{\mathbf{k}_{j}+\mathbf{k}_{m}},
$$

where $p \in \mathfrak{L}$ is as in Equation (7), and $q \in \mathfrak{L}$ has the same form as $p$, but with $\beta_{j}(\in \mathbb{C})$ instead of $\alpha_{j}$. The functional $\langle\cdot, \cdot\rangle$ is sesquilinear, $\langle p, p\rangle \geq 0$, and $\overline{\langle p, q\rangle}=\langle q, p\rangle$. Elements $u, v \in \mathfrak{L}$ are said to be equivalent, if $\langle u-v, u-v\rangle=0$. By $[p]_{\mathfrak{L}}$ we denote the equivalence class which contains $p \in \mathfrak{L}$. The equivalence classes form a finite-dimensional Hilbert space $H$. The Hilbert space $H$ is said to be associated to the moment problem (1).

We now return to the case of the solvable moment problem. Consider the space $L_{\mu}^{2}$ which consists of (the equivalence classes of) complex-valued measurable functions $f$ such that $\int|f(\mathbf{t})|^{2} d \mu<\infty$. The equivalence class in $L_{\mu}^{2}$ will be denoted by $[\cdot]_{L_{\mu}^{2}}$. Denote by $T_{l}$ the following multiplication operator:

$$
T_{l} f(\mathbf{t})=t_{l} f(\mathbf{t}), \quad f \in D_{l},
$$

with the domain $D_{l}:=\left\{f(\mathbf{t}) \in L_{\mu}^{2}: t_{l} f(\mathbf{t}) \in L_{\mu}^{2}\right\}$.

Consider the associated Hilbert space $H$, defined as above. The following transformation is useful:

$$
W \sum_{j=0}^{\rho} \alpha_{j}\left[\mathbf{t}^{\mathbf{k}_{j}}\right]_{L_{\mu}^{2}}=\sum_{j=0}^{\rho} \alpha_{j}\left[\mathbf{t}^{\mathbf{k}_{j}}\right]_{\mathfrak{L}}, \quad \alpha_{j} \in \mathbb{C} .
$$

The transformation $W$ is well-defined, linear and isometric. It maps $L_{\mu ; K}^{2}:=\operatorname{Lin}\left\{\left[\mathbf{t}^{\mathbf{k}_{j}}\right]_{L_{\mu}^{2}}\right\}_{j=0}^{\rho}$ onto $H$. Let $\vec{e}_{r}:=$ $\left(\delta_{r, m}\right)_{m=1}^{n} \in \mathbb{Z}_{+}^{n}, r=1, \ldots, n$, and

$$
\Omega_{l}=\left\{j \in\{0, \ldots, \rho\}: \mathbf{k}_{j}+\vec{e}_{l} \in K\right\}, \quad l=1, \ldots, n .
$$

Observe that

$$
W T_{l} W^{-1} \sum_{j \in \Omega_{l}} \alpha_{j}\left[\mathbf{t}^{\mathbf{k}_{j}}\right]_{\mathfrak{L}}=\sum_{j \in \Omega_{l}} \alpha_{j}\left[\mathbf{t}^{\mathbf{k}_{j}+\vec{e}_{l}}\right]_{\mathfrak{L}}, \quad \alpha_{j} \in \mathbb{C} .
$$

Since the operator $W T_{l} W^{-1}$ is well defined, the following implication holds:

$$
\left(\sum_{j \in \Omega_{l}} \alpha_{j}\left[\mathbf{t}^{\mathbf{k}_{j}}\right]_{\mathfrak{L}}=0, \quad \text { for some } \alpha_{j} \in \mathbb{C}\right) \Rightarrow\left(\sum_{j \in \Omega_{l}} \alpha_{j}\left[\mathbf{t}^{\mathbf{k}_{j}+\vec{e}_{l}}\right]_{\mathfrak{L}}=0\right) \text {. }
$$

The latter implication is equivalent to the following one:

$$
\begin{gathered}
\left(\left(\sum_{j \in \Omega_{l}} \alpha_{j}\left[\mathbf{t}^{\mathbf{k}_{j}}\right]_{\mathfrak{L}},\left[\mathbf{t}^{\mathbf{k}_{m}}\right]_{\mathfrak{L}}\right)_{H}=0, \forall m \in \Omega_{l}, \quad \text { for some } \alpha_{j} \in \mathbb{C}\right) \Rightarrow \\
\left(\left(\sum_{j \in \Omega_{l}} \alpha_{j}\left[\mathbf{t}^{\mathbf{k}_{j}+\vec{e}_{l}}\right]_{\mathfrak{L}},\left[\mathbf{t}^{\mathbf{k}_{m}+\vec{e}_{l}}\right]_{\mathfrak{L}}\right)_{H}=0, \forall m \in \Omega_{l}\right)
\end{gathered}
$$

or, equivalently,

$$
\begin{gathered}
\left(\sum_{j \in \Omega_{l}} \alpha_{j} s_{\mathbf{k}_{j}+\mathbf{k}_{m}}=0, \forall m \in \Omega_{l}, \quad \text { for some } \alpha_{j} \in \mathbb{C}\right) \Rightarrow \\
\left(\sum_{j \in \Omega_{l}} \alpha_{j} s_{\mathbf{k}_{j}+\vec{e}_{l}+\mathbf{k}_{m}+\vec{e}_{l}}=0, \forall m \in \Omega_{l}\right) .
\end{gathered}
$$


Denote

$$
\Gamma_{l}=\left(s_{\mathbf{k}_{j}+\mathbf{k}_{m}}\right)_{m, j \in \Omega_{l}}, \quad \widehat{\Gamma}_{l}=\left(s_{\mathbf{k}_{j}+\vec{e}_{l}+\mathbf{k}_{m}+\vec{e}_{l}}\right)_{m, j \in \Omega_{l}}, \quad l=1,2, \ldots, n,
$$

where the indices from $\Omega_{l}$ are taken in the increasing order. We obtain the second necessary condition of the solvability:

$$
\operatorname{Ker} \Gamma_{l} \subseteq \operatorname{Ker} \widehat{\Gamma}_{l}, \quad l=1,2, \ldots, n .
$$

We summarize our results in the following theorem.

Theorem 1. Let the moment problem (1) with $\mathcal{K}=K+K$, for an admissible finite set $K$ and some $\mathcal{S}=\left(s_{\mathbf{k}}\right)_{\mathbf{k} \in \mathcal{K}}$, be given. Then conditions (8),(17) hold.

\section{The operator approach to the moment problem. The dimensional stability.}

Suppose that for an admissible finite set $K \subset \mathbb{Z}_{+}^{n}$ the moment problem (1), with $\mathcal{K}=K+K$ and some $\mathcal{S}=$ $\left(s_{\mathbf{k}}\right)_{\mathbf{k} \in \mathcal{K}}$, is given. Fix an ordering of the elements in $K$ as in Equation (6). Assume that conditions (8),(17) hold.

We may construct the associated Hilbert space $H$, as in the previous section. For $l=1, \ldots, n$ we consider the following operators:

$$
M_{l} \sum_{j \in \Omega_{l}} \alpha_{j}\left[\mathbf{t}^{\mathbf{k}_{j}}\right]_{\mathfrak{L}}=\sum_{j \in \Omega_{l}} \alpha_{j}\left[\mathbf{t}^{\mathbf{k}_{j}+\vec{e}_{l}}\right]_{\mathfrak{L}}, \quad \alpha_{j} \in \mathbb{C},
$$

with $D\left(M_{l}\right)=\operatorname{Lin}\left\{\left[\mathbf{t}^{\mathbf{k}_{j}}\right]_{\mathfrak{L}}\right\}_{j \in \Omega_{l}}$. By condition (17) the operator $M_{l}$ is well-defined. Moreover, it is linear and symmetric. In particular, we have

$$
M_{l}\left[\mathbf{t}^{\mathbf{k}_{j}}\right]_{\mathfrak{L}}=\left[\mathbf{t}^{\mathbf{k}_{j}+\vec{e}_{l}}\right]_{\mathfrak{L}}, \quad j \in \Omega_{l} ; \quad l=1, \ldots, n .
$$

Operators $M_{l}$ are said to be associated to the moment problem (1).

Proposition 1. Let the moment problem (1) with $\mathcal{K}=K+K$, for an admissible finite set $K$ and some $\mathcal{S}=\left(s_{\mathbf{k}}\right)_{\mathbf{k} \in \mathcal{K}}$, be given and conditions (8),(17) hold. Suppose that there exist commuting self-adjoint operators $\widetilde{M}_{j} \supseteq M_{j}(j=$ $1, \ldots, n)$ in a finite-dimensional Hilbert space $\widetilde{H} \supseteq H$. Then the moment problem (1) has a solution.

Proof. Assume the existence of commuting self-adjoint operators $\widetilde{M}_{j} \supseteq M_{j}(j=1, \ldots, n)$ in a finitedimensional Hilbert space $\widetilde{H} \supseteq H$. Observe that in this case operators $\widetilde{M}_{j}$ are bounded and defined on the whole space $\widetilde{H}$. Choose an arbitrary $\mathbf{k}=\left(k_{1}, \ldots, k_{n}\right) \in K \backslash\{\mathbf{0}\}$. We shall use the notations from Definition 1. Using induction one can verify that

$$
\left[\mathbf{t}^{\widetilde{\mathbf{k}}_{r}}\right]_{\mathfrak{L}}=\widetilde{M}_{a_{r}} \ldots \widetilde{M}_{a_{1}}[1]_{\mathfrak{L}}, \quad r=1,2, \ldots,|\mathbf{k}| .
$$

In particular, we obtain that

$$
\left[\mathbf{t}^{\mathbf{k}}\right]_{\mathfrak{L}}=\widetilde{M}_{a_{|\mathbf{k}|}} \ldots \widetilde{M}_{a_{1}}[1]_{\mathfrak{L}}
$$

Since the operators $\widetilde{M}_{j}$ commute, we may rearrange the product in (21). Clearly, the operator $W_{i}$ appears $k_{i}$ times in (3). Thus, we get

$$
\left[\mathbf{t}^{\mathbf{k}}\right]_{\mathfrak{L}}=\widetilde{M}_{1}^{k_{1}} \widetilde{M}_{2}^{k_{2}} \ldots \widetilde{M}_{n}^{k_{n}}[1]_{\mathfrak{L}}, \quad \forall \mathbf{k}=\left(k_{1}, \ldots, k_{n}\right) \in K
$$

We can now construct a solution to the moment problem. For an arbitrary $\mathbf{k}=\left(k_{1}, \ldots, k_{n}\right) \in(K+K), \mathbf{k}=$ $\mathbf{k}^{\prime}+\mathbf{k}^{\prime \prime}, \mathbf{k}^{\prime}=\left(k_{1}^{\prime}, \ldots, k_{n}^{\prime}\right), \mathbf{k}^{\prime \prime}=\left(k_{1}^{\prime \prime}, \ldots, k_{n}^{\prime \prime}\right) \in K$, we may write

$$
s_{\mathbf{k}}=\left(\left[\mathbf{t}^{\mathbf{k}^{\prime}}\right],\left[\mathbf{t}^{\mathbf{k}^{\prime \prime}}\right]\right)_{H}=\left(\widetilde{M}_{1}^{k_{1}^{\prime}} \ldots \widetilde{M}_{n}^{k_{n}^{\prime}}[1]_{\mathfrak{L}}, \widetilde{M}_{1}^{k_{1}^{\prime \prime}} \ldots \widetilde{M}_{n}^{k_{n}^{\prime \prime}}[1]_{\mathfrak{L}}\right)_{H}=
$$




$$
=\left(\widetilde{M}_{1}^{k_{1}} \ldots \widetilde{M}_{n}^{k_{n}}[1]_{\mathfrak{L}},[1]_{\mathfrak{L}}\right)_{H}=\int \mathbf{t}^{\mathbf{k}} d \mu(\mathbf{t}),
$$

where

$$
\mu(\delta)=\left(E(\delta)[1]_{\mathfrak{L}},[1]_{\mathfrak{L}}\right)_{H}, \quad \delta \in \mathfrak{B}\left(\mathbb{R}^{n}\right),
$$

where $E(\delta)$ is the spectral measure of a commuting tuple $\widetilde{M}_{1}, \ldots, \widetilde{M}_{n}$. Consequently, we get a solution $\mu$ of the moment problem.

We now present some explicit numerical conditions which ensure that the associated operator $M_{l}$ is selfadjoint. Denote

$$
\begin{gathered}
g_{j}:=\left[\mathbf{t}^{\mathbf{k}_{j}}\right]_{\mathfrak{L}}, \quad j \in \mathbb{Z}_{0, \rho}, \\
\Omega_{l}^{\prime}:=\{0, \ldots, \rho\} \backslash \Omega_{l}, \quad l=1, \ldots, n .
\end{gathered}
$$

Observe that

$$
\left(g_{j}, g_{m}\right)_{H}=s_{\mathbf{k}_{j}+\mathbf{k}_{m}}, \quad j, m \in \mathbb{Z}_{0, \rho} .
$$

Theorem 2. Let the moment problem (1) with $\mathcal{K}=K+K$, for an admissible finite set $K$ and some $\mathcal{S}=\left(s_{\mathbf{k}}\right)_{\mathbf{k} \in \mathcal{K}}$, be given and conditions (8),(17) hold. Fix an arbitrary $l \in \mathbb{Z}_{1, n}$. For every $j \in \Omega_{l}^{\prime}$, denote by $\alpha_{t}(j)\left(t \in \Omega_{l}\right)$ an arbitrary complex solution of the following linear algebraic system:

$$
\sum_{t \in \Omega_{l}} \alpha_{t}(j) s_{\mathbf{k}_{t}+\mathbf{k}_{k}}=s_{\mathbf{k}_{j}+\mathbf{k}_{k}}, \quad k \in \Omega_{l} .
$$

The associated operator $M_{l}$ is self-adjoint if and only if for every $j \in \Omega_{l}^{\prime}$ the following relation holds:

$$
\sum_{t, r \in \Omega_{l}} \alpha_{t}(j) \overline{\alpha_{r}(j)} s_{\mathbf{k}_{t}+\mathbf{k}_{r}}-2 \operatorname{Re}\left(\sum_{t \in \Omega_{l}} \alpha_{t}(j) s_{\mathbf{k}_{t}+\mathbf{k}_{j}}\right)+s_{\mathbf{k}_{j}+\mathbf{k}_{j}}=0 .
$$

Proof. The operator $M_{l}$ is self-adjoint if and only if

$$
g_{j} \in D\left(M_{l}\right), \quad j \in \Omega_{l}^{\prime} .
$$

In fact, in the latter case the operator $M_{l}$ is symmetric and defined on the whole finite-dimensional space $H$. We shall give a simple but general argument. It will be also used later. Let $h$ be an arbitrary vector from $H$, and $G:=\operatorname{Lin}\left\{g_{k}\right\}_{k \in \widetilde{\Omega}}$, where $\widetilde{\Omega}$ is an arbitrary subset of $\{0, \ldots, \rho\}$. We denote by $y$ the orthogonal projection of $h$ onto $G$. Then

$$
\left(h, g_{k}\right)_{H}=\left(y, g_{k}\right)_{H}, \quad k \in \widetilde{\Omega} .
$$

Since $y$ belongs to $G$, it has the following form: $y=\sum_{t \in \widetilde{\Omega}} \alpha_{t} g_{t}, \alpha_{t} \in \mathbb{C}$. Then

$$
\sum_{t \in \widetilde{\Omega}} \alpha_{t}\left(g_{t}, g_{k}\right)_{H}=\left(h, g_{k}\right)_{H}, \quad k \in \widetilde{\Omega} .
$$

Conversely, let $\alpha_{t} \in \mathbb{C}(t \in \widetilde{\Omega})$ be an arbitrary solution of the linear system (30). Set $\widehat{y}:=\sum_{t \in \widetilde{\Omega}} \alpha_{t} g_{t}$. By (30) we conclude that

$$
\left(\widehat{y}, g_{k}\right)_{H}=\left(h, g_{k}\right)_{H}, \quad k \in \widetilde{\Omega} \text {. }
$$

Therefore $h-\widehat{y} \perp G$, and $\widehat{y}=y$.

Applying the above argument to the case $h=g_{j}\left(j \in \Omega_{l}^{\prime}\right)$ and $\widetilde{\Omega}=\Omega_{l}$, we conclude that $y_{j}:=\sum_{t \in \Omega_{l}} \alpha_{t}(j) g_{t}$ is the projection of $g_{j}$ onto $D\left(M_{l}\right)$. Condition (29) is equivalent to the following condition:

$$
\left\|y_{j}-g_{j}\right\|^{2}=\left(y_{j}, y_{j}\right)-\left(y_{j}, g_{j}\right)-\left(g_{j}, y_{j}\right)+\left(g_{j}, g_{j}\right)=0, \quad j \in \Omega_{l}^{\prime} \text {. }
$$

The latter condition can be rewritten in the form (28). 
Suppose that for the associated operator $M_{l}\left(l \in \mathbb{Z}_{1, n}\right)$ conditions of Theorem 2 hold. From the proof of Theorem 2 it is clear that

$$
g_{j}=\sum_{t \in \Omega_{l}} \alpha_{t}(j) g_{t}, \quad j \in \Omega_{l}^{\prime}
$$

Therefore

$$
M_{l} g_{j}=\sum_{t \in \Omega_{l}} \alpha_{t}(j) M_{l} g_{t}=\sum_{t \in \Omega_{l}} \alpha_{t}(j)\left[\mathbf{t}^{\mathbf{k}_{t}+\vec{e}_{l}}\right]_{\mathfrak{L}}, \quad j \in \Omega_{l}^{\prime} .
$$

Thus, by relations (19),(33) the operator $M_{l}$ is explicitly defined on each vector $g_{j}, j=0, \ldots, \rho$. We shall rewrite this in the following form:

$$
M_{l} g_{j}=\sum_{k=0}^{\rho} \widehat{\alpha}_{k}(j) g_{k}, \quad j \in\{0, \ldots, \rho\},
$$

with complex coefficients $\widehat{\alpha}_{k}(j)$ (by gathering the coefficients by each $g_{k}$ in the right-hand sides of relations (19),(33)).

We now assume additionally that for the associated operator $M_{r}\left(r \in \mathbb{Z}_{1, n}\right)$ conditions of Theorem 2 hold, as well. Then

$$
M_{r} g_{j}=\sum_{k=0}^{\rho} \widehat{\beta}_{k}(j) g_{k}, \quad j \in\{0, \ldots, \rho\},
$$

with some complex $\widehat{\beta}_{k}(j)$. By (34) and (35) we come to the following relation:

$$
\left(M_{l} M_{r}-M_{r} M_{l}\right) g_{j}=\sum_{k=0}^{\rho} \gamma_{k}(j) g_{k}, \quad j \in\{0, \ldots, \rho\},
$$

with some complex $\gamma_{k}(j)$.

Theorem 3. Let the moment problem (1) with $\mathcal{K}=K+K$, for an admissible finite set $K$ and some $\mathcal{S}=\left(s_{\mathbf{k}}\right)_{\mathbf{k} \in \mathcal{K}}$, be given and conditions (8),(17) hold. Suppose that for the associated operators $M_{l}, M_{r}\left(l, r \in \mathbb{Z}_{1, n}\right)$ conditions of Theorem 2 hold. Define coefficients $\gamma_{k}(j), j, k \in \mathbb{Z}_{0, \rho}$, as in Relation (36). The operators $M_{l}$ and $M_{r}$ commute if and only if the following relation holds:

$$
\sum_{k, t=0}^{\rho} \gamma_{k}(j) \overline{\gamma_{t}(j)} s_{\mathbf{k}_{k}+\mathbf{k}_{t}}=0, \quad j \in \mathbb{Z}_{0, \rho} .
$$

Proof. It follows from the preceding arguments.

Definition 2. Suppose that for an admissible finite set $K \subset \mathbb{Z}_{+}^{n}$ the moment problem (1), with $\mathcal{K}=K+K$ and some $\mathcal{S}=\left(s_{\mathbf{k}}\right)_{\mathbf{k} \in \mathcal{K}}$, is given and conditions (8),(17) hold. Define the associated Hilbert space $H$ and the associated operators $M_{l}(l=1, \ldots, n)$. The set of moments $\mathcal{S}$ is said to be completely self-adjoint, if the operators $M_{l}$ are self-adjoint and pairwise commute.

Theorem 4. Let the moment problem (1) with $\mathcal{K}=K+K$, for an admissible finite set $K$ and some $\mathcal{S}=\left(s_{\mathbf{k}}\right)_{\mathbf{k} \in \mathcal{K}}$, be given and conditions (8),(17) hold. The set of moments $S$ is completely self-adjoint, if and only if the following conditions hold:

(a) For the associated operators $M_{l}\left(l \in \mathbb{Z}_{1, n}\right)$ conditions of Theorem 2 hold.

(b) For the operators $M_{l}, M_{r}$ conditions of Theorem 3 hold, for all $l, r \in \mathbb{Z}_{1, n}: l<r$.

If conditions ( $a),(b)$ hold, then the moment problem (1) has a solution.

Proof. The statement about conditions $(a),(b)$ follows, if we apply Definition 2 and Theorems 2 and 3. The last statement of the Theorem follows by Proposition 1. 
Denote

$$
\Omega_{0}=\left\{j \in\{0, \ldots, \rho\}: \mathbf{k}_{j}+\vec{e}_{1}, \mathbf{k}_{j}+\vec{e}_{2}, \ldots, \mathbf{k}_{j}+\vec{e}_{n} \in K\right\},
$$

and

$$
H_{0}=\operatorname{Lin}\left\{\left[\mathbf{t}^{\mathbf{k}_{j}}\right]_{\mathfrak{L}}\right\}_{j \in \Omega_{0}} .
$$

Observe that

$$
\Omega_{0} \subseteq \Omega_{j}, \quad j=1, \ldots, n,
$$

and therefore

$$
H_{0} \subseteq D\left(M_{j}\right), \quad j=1, \ldots, n
$$

Definition 3. Suppose that for an admissible finite set $K \subset \mathbb{Z}_{+}^{n}$ the moment problem (1), with $\mathcal{K}=K+K$ and some $\mathcal{S}=\left(s_{\mathbf{k}}\right)_{\mathbf{k} \in \mathcal{K}}$, is given and conditions (8),(17) hold. Define the associated Hilbert space $H$ and its subspace $H_{0}$. The set of moments $\mathcal{S}$ is said to be dimensionally stable, if $\operatorname{dim} H=\operatorname{dim} H_{0}$.

The dimensional stability can be verified explicitly by the given moments. Denote

$$
\Omega_{0}^{\prime}:=\{0, \ldots, \rho\} \backslash \Omega_{0} .
$$

Theorem 5. Let the moment problem (1) with $\mathcal{K}=K+K$, for an admissible finite set $K$ and some $\mathcal{S}=\left(s_{\mathbf{k}}\right)_{\mathbf{k} \in \mathcal{K}}$, be given and conditions (8),(17) hold. For every $j \in \Omega_{0}^{\prime}$, denote by $\alpha_{t}(j)\left(t \in \Omega_{0}\right)$ an arbitrary complex solution of the following linear algebraic system:

$$
\sum_{t \in \Omega_{0}} \alpha_{t}(j) s_{\mathbf{k}_{t}+\mathbf{k}_{k}}=s_{\mathbf{k}_{j}+\mathbf{k}_{k}}, \quad k \in \Omega_{0}
$$

The set of moments $\mathcal{S}$ is dimensionally stable, if and only if the following relation holds:

$$
\sum_{t, r \in \Omega_{0}} \alpha_{t}(j) \overline{\alpha_{r}(j)} s_{\mathbf{k}_{t}+\mathbf{k}_{r}}-2 \operatorname{Re}\left(\sum_{t \in \Omega_{0}} \alpha_{t}(j) s_{\mathbf{k}_{t}+\mathbf{k}_{j}}\right)+s_{\mathbf{k}_{j}+\mathbf{k}_{j}}=0, \forall j \in \Omega_{0}^{\prime} .
$$

Proof. Observe that the set of moments $\mathcal{S}$ is dimensionally stable, if and only if

$$
g_{j} \in H_{0}, \quad j \in \Omega^{\prime} .
$$

Denote by $y_{j}$ the projection of the vector $g_{j}\left(j \in \Omega^{\prime}\right)$ onto $H_{0}$. By the general argument, presented in the proof of Theorem 2, we conclude that

$$
y_{j}=\sum_{t \in \Omega_{0}} \alpha_{t}(j) g_{t}
$$

It remains to notice that relation (42) is equivalent to the following relation:

$$
\left(y_{j}-g_{j}, y_{j}-g_{j}\right)=0, \quad j \in \Omega^{\prime},
$$

which can be written as in (41).

Suppose that for the moment problem, as in Definition 3, the set $\mathcal{S}$ is dimensionally stable. Then operators $M_{l}$ are self-adjoint and defined on the whole $H$. Observe that for $l, r \in\{1, \ldots, n\}: l \neq r$, we have

$$
M_{l} M_{r}\left[\mathbf{t}^{\mathbf{k}_{j}}\right]_{\mathfrak{L}}=M_{l}\left[\mathbf{t}^{\mathbf{k}_{j}+\vec{e}_{r}}\right]_{\mathfrak{L}}, \quad \forall j \in \Omega_{0} .
$$

In general, it is not clear if the element $\mathbf{k}_{j}+\vec{e}_{r}=: \mathbf{k}_{s}$, with $s \in\{0, \ldots, \rho\}$, has the property $s \in \Omega_{l}$. Thus, we can not apply relation (19) to get $\left[\mathbf{t}^{\mathbf{k}_{j}+\vec{e}_{r}+\vec{e}_{l}}\right]_{\mathfrak{L}}$. However, the following theorem holds.

Theorem 6. Let the moment problem (1) with $\mathcal{K}=K+K$, for a set $K$ as in Relation (5) and some $\mathcal{S}=\left(s_{\mathbf{k}}\right)_{\mathbf{k} \in \mathcal{K}}$, be given. Suppose that conditions (8),(17) hold and that $\mathcal{S}$ is dimensionally stable. Then $\mathcal{S}$ is completely self-adjoint and the moment problem (1) has a solution. 
Proof. In fact, for the type of truncations as in (5), when applying the operator $M_{r}$ in (43) we do not leave the domain of the operator $M_{l}$. Therefore we obtain that

$$
M_{l} M_{r}\left[\mathbf{t}^{\mathbf{k}_{j}}\right]_{\mathfrak{L}}=\left[\mathbf{t}^{\mathbf{k}_{j}+\vec{e}_{r}+\vec{e}_{l}}\right]_{\mathfrak{L}}=M_{r} M_{l}\left[\mathbf{t}^{\mathbf{k}_{j}}\right]_{\mathfrak{L}} .
$$

Thus, we have the completely self-adjoint case. It remains to apply Theorem 4.

Thus, in the case of rectangular truncations the dimensional stability (DS) implies the complete selfadjointness (CS):

$$
(D S) \Rightarrow(C S) \text {. }
$$

It is not clear if implication (44) holds for any admissible truncations. The validity of the inverse implication in (44) is also of interest.

Suppose that for an admissible finite set $K \subset \mathbb{Z}_{+}^{n}$ the moment problem (1), with $\mathcal{K}=K+K$ and some $\mathcal{S}=\left(s_{\mathbf{k}}\right)_{\mathbf{k} \in \mathcal{K}}$, is given and conditions (8),(17) hold. Define the associated Hilbert space $H$ and its subspace $H_{0}$. To verify the dimensional stability, one can use Theorem 5 . On the other hand, one can find projections of elements $g_{j}\left(j \in \Omega_{0}^{\prime}\right)$ on the subspace $H_{0}$, by using an orthonormal basis in $H_{0}$.

Example 2. Consider the truncated moment problem (1) with $n=2, K=K_{2,2}$ (see Example 1$), \mathcal{K}=K+K=K_{4,4}$, and the following moments:

$$
\begin{aligned}
& s_{(0,0)}=3, s_{(0,1)}=s_{(0,2)}=s_{(0,3)}=s_{(0,4)}=1, \\
& s_{(1,0)}=4, s_{(1,1)}=s_{(1,2)}=s_{(1,3)}=s_{(1,4)}=0, \\
& s_{(2,0)}=4, s_{(2,1)}=s_{(2,2)}=s_{(2,3)}=s_{(2,4)}=0, \\
& s_{(3,0)}=16, s_{(3,1)}=s_{(3,2)}=s_{(3,3)}=s_{(3,4)}=0, \\
& s_{(4,0)}=32, s_{(4,1)}=s_{(4,2)}=s_{(4,3)}=s_{(4,4)}=0 .
\end{aligned}
$$

Order the elements of $K$ as follows:

$$
\begin{aligned}
& \mathbf{k}_{0}=(0,0), \mathbf{k}_{1}=(0,1), \mathbf{k}_{2}=(0,2), \\
& \mathbf{k}_{3}=(1,0), \mathbf{k}_{4}=(1,1), \mathbf{k}_{5}=(1,2), \\
& \mathbf{k}_{6}=(2,0), \mathbf{k}_{7}=(2,1), \mathbf{k}_{8}=(2,2) .
\end{aligned}
$$

Thus, we have $\rho=8$. The matrix $\Gamma=\left(s_{\mathbf{k}_{j}+\mathbf{k}_{m}}\right)_{j, m=0}^{8}$ has the following form:

$$
\Gamma=\left(\begin{array}{ccccccccc}
3 & 1 & 1 & 4 & 0 & 0 & 8 & 0 & 0 \\
1 & 1 & 1 & 0 & 0 & 0 & 0 & 0 & 0 \\
1 & 1 & 1 & 0 & 0 & 0 & 0 & 0 & 0 \\
4 & 0 & 0 & 8 & 0 & 0 & 16 & 0 & 0 \\
0 & 0 & 0 & 0 & 0 & 0 & 0 & 0 & 0 \\
0 & 0 & 0 & 0 & 0 & 0 & 0 & 0 & 0 \\
8 & 0 & 0 & 16 & 0 & 0 & 32 & 0 & 0 \\
0 & 0 & 0 & 0 & 0 & 0 & 0 & 0 & 0 \\
0 & 0 & 0 & 0 & 0 & 0 & 0 & 0 & 0
\end{array}\right) .
$$

The non-negativity of $\Gamma$ can be verified directly, by checking that the determinants of all submatrices, standing on the intersections of rows and columns with the same indices, are non-negative. The matrices $\Gamma_{1}, \Gamma_{2}, \widehat{\Gamma}_{1}, \widehat{\Gamma}_{2}$ have the following forms:

$$
\Gamma_{1}=\left(\begin{array}{cccccc}
3 & 1 & 1 & 4 & 0 & 0 \\
1 & 1 & 1 & 0 & 0 & 0 \\
1 & 1 & 1 & 0 & 0 & 0 \\
4 & 0 & 0 & 8 & 0 & 0 \\
0 & 0 & 0 & 0 & 0 & 0 \\
0 & 0 & 0 & 0 & 0 & 0
\end{array}\right), \quad \Gamma_{2}=\left(\begin{array}{cccccc}
3 & 1 & 4 & 0 & 8 & 0 \\
1 & 1 & 0 & 0 & 0 & 0 \\
4 & 0 & 8 & 0 & 16 & 0 \\
0 & 0 & 0 & 0 & 0 & 0 \\
8 & 0 & 16 & 0 & 32 & 0 \\
0 & 0 & 0 & 0 & 0 & 0
\end{array}\right),
$$




$$
\widehat{\Gamma}_{1}=\left(\begin{array}{cccccc}
8 & 0 & 0 & 16 & 0 & 0 \\
0 & 0 & 0 & 0 & 0 & 0 \\
0 & 0 & 0 & 0 & 0 & 0 \\
16 & 0 & 0 & 32 & 0 & 0 \\
0 & 0 & 0 & 0 & 0 & 0 \\
0 & 0 & 0 & 0 & 0 & 0
\end{array}\right), \quad \widehat{\Gamma}_{2}=\left(\begin{array}{cccccc}
1 & 1 & 0 & 0 & 0 & 0 \\
1 & 1 & 0 & 0 & 0 & 0 \\
0 & 0 & 0 & 0 & 0 & 0 \\
0 & 0 & 0 & 0 & 0 & 0 \\
0 & 0 & 0 & 0 & 0 & 0 \\
0 & 0 & 0 & 0 & 0 & 0
\end{array}\right) .
$$

The linear algebraic equation

$$
\Gamma_{1} \vec{x}=0, \quad \vec{x}=\left(x_{1}, \ldots, x_{6}\right)^{T},
$$

has the following solution: $x_{3}, x_{4}, x_{5}, x_{6}$ are arbitrary complex numbers, $x_{1}=-2 x_{4}, x_{2}=-x_{3}+2 x_{4}$. It is easy to verify that any solution satisfies $\widehat{\Gamma}_{1} \vec{x}=0$.

On the other hand, the linear algebraic equation

$$
\Gamma_{2} \vec{x}=0, \quad \vec{x}=\left(x_{1}, \ldots, x_{6}\right)^{T},
$$

has the following solution: $x_{2}, x_{4}, x_{5}, x_{6}$ are arbitrary complex numbers, $x_{1}=-x_{2}, x_{3}=\frac{1}{2} x_{2}-2 x_{5}$. Again, one can verify that any solution satisfies $\widehat{\Gamma}_{2} \vec{x}=0$.

Thus, conditions (8),(17) hold. Let us check the dimensional stability. Consider the associated Hilbert space H. For simplicity, we denote

$$
g_{j}=\left[\mathbf{t}^{\mathbf{k}_{j}}\right]_{\mathfrak{L}}, \quad j=0, \ldots, 8 .
$$

Observe that

$$
\Omega_{0}=\{0,1,3,4\} .
$$

Let us apply the Gram-Schmidt orthogonalization process, removing linearly dependent elements, to the sequence $g_{0}, g_{1}, g_{3}, g_{4}$. Notice that all norms and scalar products are calculated by the moments:

$$
\left(g_{j}, g_{r}\right)_{H}=\left(\left[\mathbf{t}^{\mathbf{k}_{j}}\right]_{\mathfrak{L}},\left[\mathbf{t}^{\mathbf{k}_{r}}\right]_{\mathfrak{L}}\right)_{H}=s_{\mathbf{k}_{j}+s_{\mathbf{k}_{r}}}, \quad j, r=0,1, \ldots, 8 .
$$

We obtain an orthonormal basis $\mathfrak{F}=\left\{f_{0}, f_{1}\right\}$ in $H_{0}$, with

$$
f_{0}=\frac{1}{\sqrt{3}} g_{0}, \quad f_{1}=\sqrt{\frac{3}{2}}\left(g_{1}-\frac{1}{3} g_{0}\right) .
$$

Moreover, it turned out that

$$
g_{3}=2 g_{0}-2 g_{1}, \quad g_{4}=0 .
$$

It remains to verify that the projections of elements $g_{2}, g_{5}, g_{6}, g_{7}, g_{8}$ on $H_{0}$ coincide with the corresponding elements. For example,

$$
g_{2}-\left(g_{2}, f_{0}\right) f_{0}-\left(g_{2}, f_{1}\right) f_{1}=g_{2}-g_{1}
$$

but

$$
\left\|g_{2}-g_{1}\right\|_{H}^{2}=\left(g_{2}-g_{1}, g_{2}-g_{1}\right)_{H}=\left(g_{2}, g_{2}\right)-\left(g_{2}, g_{1}\right)-\left(g_{1}, g_{2}\right)+\left(g_{1}, g_{1}\right)=0 \text {. }
$$

For other elements, we proceed in a similar way. Consequently, the sequence $\mathcal{S}=\left(s_{\mathbf{k}}\right)_{\mathbf{k} \in \mathcal{K}}$ is dimensionally stable.

Let us construct an atomic solution $\mu$ of the moment problem. Observe that

$$
\Omega_{1}=\{0,1,2,3,4,5\}, \quad \Omega_{2}=\{0,1,3,4,6,7\} .
$$

The operators $M_{1}$ and $M_{2}$ act in the following way:

$$
\begin{gathered}
M_{1} g_{0}=g_{3}=2 g_{0}-g_{1}, \quad M_{1} g_{1}=g_{4}=0 ; \\
M_{2} g_{0}=g_{1}, \quad M_{2} g_{1}=g_{2}=g_{1} .
\end{gathered}
$$

Therefore

$$
M_{1} f_{0}=\frac{4}{3} f_{0}-\frac{2 \sqrt{2}}{3} f_{1}, \quad M_{1} f_{1}=-\frac{2 \sqrt{2}}{3} f_{0}+\frac{2}{3} f_{1}
$$




$$
M_{2} f_{0}=\frac{1}{3} f_{0}+\frac{\sqrt{2}}{3} f_{1}, \quad M_{1} f_{1}=\frac{\sqrt{2}}{3} f_{0}+\frac{2}{3} f_{1} .
$$

The matrices $\mathcal{M}_{1}, \mathcal{M}_{2}$ of operators $M_{1}, M_{2}$, respectively, for the basis $\mathfrak{F}$ are:

$$
\mathcal{M}_{1}=\left(\begin{array}{cc}
\frac{4}{3} & -\frac{2 \sqrt{2}}{3} \\
-\frac{2 \sqrt{2}}{3} & \frac{2}{3}
\end{array}\right), \quad \mathcal{M}_{2}=\left(\begin{array}{cc}
\frac{1}{3} & \frac{\sqrt{2}}{3} \\
\frac{\sqrt{2}}{3} & \frac{2}{3}
\end{array}\right) .
$$

The matrix $\mathcal{M}_{1}$ has eigenvalues $\lambda_{1}=0, \lambda_{2}=2$, with eigenvectors respectively:

$$
\vec{u}_{1}=\frac{1}{\sqrt{3}}(1, \sqrt{2})^{T}, \quad \vec{u}_{2}=\frac{1}{\sqrt{3}}(-\sqrt{2}, 1)^{T} .
$$

The matrix $\mathcal{M}_{2}$ has eigenvalues $\widetilde{\lambda}_{1}=0, \widetilde{\lambda}_{2}=1$, with eigenvectors respectively:

$$
\vec{v}_{1}=\frac{1}{\sqrt{3}}(\sqrt{2},-1)^{T}, \quad \vec{v}_{2}=\frac{1}{\sqrt{3}}(1, \sqrt{2})^{T} .
$$

Let

$$
\begin{array}{ll}
\mathcal{H}_{1}=\operatorname{Lin}\left\{\frac{1}{\sqrt{3}}\left(f_{0}+\sqrt{2} f_{1}\right)\right\}, & \mathcal{H}_{2}=\operatorname{Lin}\left\{\frac{1}{\sqrt{3}}\left(-\sqrt{2} f_{0}+f_{1}\right)\right\}, \\
\widetilde{\mathcal{H}}_{1}=\operatorname{Lin}\left\{\frac{1}{\sqrt{3}}\left(\sqrt{2} f_{0}-f_{1}\right)\right\}, & \widetilde{\mathcal{H}}_{2}=\operatorname{Lin}\left\{\frac{1}{\sqrt{3}}\left(f_{0}+\sqrt{2} f_{1}\right)\right\} .
\end{array}
$$

Observe that the spectral measure $E(\delta)$ in relation (24) can have jumps at points $(x, y)$ with $x \in\left\{\lambda_{1}, \lambda_{2}\right\}, y \in$ $\left\{\widetilde{\lambda}_{1}, \widetilde{\lambda}_{2}\right\}$. The measure support is contained in this set of four points. Thus, the measure $\mu$ has at most 4 atoms. Notice that

$$
\begin{gathered}
\mu(\{(x, y)\})=\left(E(\{(x, y)\}) g_{0}, g_{0}\right)_{H}=\left(E_{1}(\{x\}) E_{2}(\{y\}) g_{0}, g_{0}\right)_{H}= \\
=\left(E_{2}(\{y\}) g_{0}, E_{1}(\{x\}) g_{0}\right)_{H}, \quad \forall(x, y) \in \mathbb{R}^{2} .
\end{gathered}
$$

Observe that

$$
\begin{aligned}
& E_{1}(\{0\}) g_{0}=P_{\mathcal{H}_{1}} g_{0}=\frac{1}{\sqrt{3}}\left(f_{0}+\sqrt{2} f_{1}\right), \\
& E_{1}(\{2\}) g_{0}=P_{\mathcal{H}_{2}} g_{0}=\frac{\sqrt{6}}{3}\left(\sqrt{2} f_{0}-f_{1}\right), \\
& E_{2}(\{0\}) g_{0}=P_{\widetilde{\mathcal{H}}_{1}} g_{0}=\frac{\sqrt{6}}{3}\left(\sqrt{2} f_{0}-f_{1}\right), \\
& E_{2}(\{1\}) g_{0}=P_{\widetilde{\mathcal{H}}_{2}} g_{0}=\frac{1}{\sqrt{3}}\left(f_{0}+\sqrt{2} f_{1}\right) .
\end{aligned}
$$

By (51) we conclude that the solution $\mu$ is 2-atomic, having jumps 1 and 2 at points $(0,1)$ and $(2,0)$, respectively.

\section{An algorithm for the truncated two-dimensional moment problem.}

In this section we shall study the case $n=2$ of the moment problem (1). We shall give two algoritms: Algorithm 1 and Algorithm 2. These algorithms always give the desired output for any correct input data. Let us briefly describe them. The task of Algorithm 1 is to obtain the matrices of all self-adjoint extensions of the associated operators $M_{l}$ in the corresponding Hilbert space $H$. We emphasize that the commutativity for the extensions is not assumed. Self-adjoint extensions of any symmetric operator $A$, with equal deficiency indices, always exist. In the case of the densely defined operator it follows by von Neumann's formulas. In the general case it follows by generalized von Neumann's formulas (see, e.g., [26, Theorem 3.13]).

The input data for Algorithm 2 includes the matrices of two commuting self-adjoint extensions $\widetilde{M}_{l} \supseteq M_{l}$ in $H$. The output is a solution of the moment problem (1). Thus, the moment problem reduces to providing a 
link between the algorithms. One should extract in some way commuting extensions between those given by Algorithm 1. Sufficient conditions for such a successful extraction will be given in Theorem 7 .

If $s_{\mathbf{0}}=0$, then the moment problem (1) can not have any solution different from $\mu=0$. In this case, if all the moments are zero then $\mu=0$ is a solution, otherwise there are no solutions. Thus, we can exclude the case $s_{\mathbf{0}}=0$ from our further considerations.

Algorithm 1 (The construction of all self-adjoint extensions of $M_{l}$ in $H$ ).

Input: an admissible finite set $K \subset \mathbb{Z}_{+}^{2}, \mathcal{K}:=K+K$ and a set of prescribed moments $\mathcal{S}=\left(s_{\mathbf{k}}\right)_{\mathbf{k} \in \mathcal{K}}$, with $s_{\mathbf{0}} \neq 0$, which satisfies the necessary conditions (8) and (17). Here we fix an ordering of the elements in $K$ as in Equation (6), with $\mathbf{k}_{0}=\mathbf{0}$.

Step 1. Consider the associated Hilbert space $H$, which is defined as in the paragraph following formula (8). We shall use the brief notation (25). Although this space consists of abstract elements (the equivalence classes), all numerical calculations will be performed by the basic property (26). For $l=1,2$ we consider the associated operators $M_{l}$ (see (18)). Observe that

$$
M_{l} g_{j}=\left[\mathbf{t}^{\mathbf{k}_{j}+\vec{e}_{l}}\right]_{\mathfrak{L}}=: g_{\eta(l ; j)}, \quad j \in \Omega_{l} ; \quad l=1,2 .
$$

Step 2. (The construction of an orthonormal basis in $H$ ).

Apply the Gram-Schmidt orthogonalization procedure to the sequence

$$
g_{0}, g_{1}, \ldots, g_{\rho},
$$

removing the linearly dependent elements, if they appear. We get an orthonormal basis

$$
\mathfrak{F}=\left\{f_{0}, f_{1}, \ldots, f_{\rho^{\prime}}\right\}
$$

in the Hilbert space $H$ (where $0 \leq \rho^{\prime} \leq \rho$ ). By the construction, an element $f_{j}$ is a linear combination of $g_{k} \mathrm{~s}$, with explicitly calculated coefficients. Notice that $f_{0} \neq 0$.

Step 3. (The parametrization of all linear extensions of $M_{l}$ ).

Observe that $M_{l}$ is defined on elements $g_{j}, j \in \Omega_{l}(l=1,2)$. At first, define linear operators $\widetilde{M}_{l}$ on these elements in the same way. Denote

$$
\Omega_{l}^{\prime}:=\{0,1, \ldots, \rho\} \backslash \Omega_{l}, \quad l=1,2 .
$$

For $l=1,2$ one should repeat the following procedure.

Choose an arbitrary element $g_{k}, k \in \Omega_{l}^{\prime}$. Calculate the norm of its projection on $D\left(\widetilde{M}_{l}\right)$. If $g_{k} \in D\left(\widetilde{M}_{l}\right)$, then we skip this element. Otherwise, we set

$$
\widetilde{M}_{l} g_{k}:=\sum_{j=0}^{\rho^{\prime}}\left(\alpha_{l ; k, j}+\beta_{l ; k, j} i\right) f_{j}, \quad \alpha_{l ; k, j}, \beta_{l ; k, j} \in \mathbb{R},
$$

and extend the domain of $\widetilde{M}_{l}$, using linearity. Then we take another element $g_{k}, k \in \Omega_{l}^{\prime}$, and proceed in a similar way. We continue this procedure to define $\widetilde{M}_{l}$ on the whole $H$. This completes the procedure for $M_{l}$.

Notice that the case $D\left(M_{l}\right)=H$ was not excluded in the above procedure. The latter case means that the corresponding parameters $\alpha_{l ; k, j}, \beta_{l ; k, j}$ are absent.

Step 4. (The calculation of matrices of $\widetilde{M}_{l}$ ). Observe that each $f_{j}$ is a linear combination of $g_{k}$ s (by the GramSchmidt orthogonalization):

$$
f_{j}=\sum_{k=0}^{\rho} c_{j ; k} g_{k}, \quad c_{j ; k} \in \mathbb{C} ; j \in \mathbb{Z}_{0, \rho^{\prime}} ;
$$

and vice versa:

$$
g_{j}=\sum_{k=0}^{\rho^{\prime}} d_{j ; k} f_{k}, \quad d_{j ; k} \in \mathbb{C} ; j \in \mathbb{Z}_{0, \rho} .
$$

Then

$$
\widetilde{M}_{l} f_{j}=\sum_{k=0}^{\rho} c_{j ; k} \widetilde{M}_{l} g_{k}
$$


By (52), (54) and (56) we see that $\widetilde{M}_{l} f_{j}$ is a linear combination of $f_{k}$ with some coefficients, which may depend linearly on $\alpha_{l ; k, j}, \beta_{l ; k, j}$.

In the basis $\mathfrak{F}$, we calculate the matrices $\mathcal{M}_{1}, \mathcal{M}_{2}$ of $\widetilde{M}_{1}$ and $\widetilde{M}_{2}$, respectively. The coefficients of $\mathcal{M}_{1}, \mathcal{M}_{2}$ may depend linearly on real parameters $\alpha_{l ; k, j}, \beta_{l ; k, j}$.

Step 5. (The extraction of self-adjoint extensions of $M_{l}$ ).

The following condition:

$$
\mathcal{M}_{1}=\mathcal{M}_{1}^{\star}, \quad \mathcal{M}_{2}=\mathcal{M}_{2}^{\star},
$$

ensures the self-adjointness of $\widetilde{M}_{1}$ and $\widetilde{M}_{2}$. Equating the corresponding entries of matrices in (57) we obtain linear algebraic systems with complex coefficients for unknown real parameters $\alpha_{l ; k, j}, \beta_{l ; k, j}$. Taking the real and the imaginary parts of these equations, we get linear algebraic systems with real coefficients and real unknowns $\alpha_{l ; k, j}, \beta_{l ; k, j}$. They can be solved by the Gauss elimination method. The solutions should be inserted in the matrices $\mathcal{M}_{1}, \mathcal{M}_{2}$. The matrices $\mathcal{M}_{1}, \mathcal{M}_{2}$ parametrize all self-adjoint extensions of the corresponding operators in $H$.

Output: matrices $\mathcal{M}_{1}, \mathcal{M}_{2}$, which can depend on a finite number of free real parameters.

We now present some sufficient conditions, which allow to obtain a solution of the moment problem.

Theorem 7. Let the moment problem (1) with $n=2, \mathcal{K}=K+K$, for an admissible finite set $K$ and some $\mathcal{S}=\left(s_{\mathbf{k}}\right)_{\mathbf{k} \in \mathcal{K}}$, be given and conditions (8),(17) hold. Suppose that for one of the associated operators $M_{1}, M_{2}$ condition (28) of Theorem 2 holds. Consider the matrices $\mathcal{M}_{1}, \mathcal{M}_{2}$, which can depend on a finite number of free real parameters, constructed by Algorithm 1. If the following linear system of algebraic equations:

$$
\mathcal{M}_{1} \mathcal{M}_{2}=\mathcal{M}_{2} \mathcal{M}_{1},
$$

has a solution, then the moment problem (1) is solvable.

Remark 1. Observe that condition (28) of Theorem 2 ensures that one of the associated operators is self-adjoint. Therefore one of the matrices $\mathcal{M}_{1}, \mathcal{M}_{2}$ has no parameters. Equating the corresponding entries of matrices in (58), we obtain a linear algebraic system with complex coefficients and real unknowns. Taking the real and the imaginary parts of these equations we get a linear algebraic system with real coefficients and real unknowns, which can be solved by the Gauss elimination method.

Proof. By Remark 1 we conclude that the operators $M_{1}$ and $M_{2}$ have commuting self-adjoint extensions in $H$. Applying Proposition 1 we obtain that the moment problem has a solution.

The following algorithm provides a solution to the moment problem, if we have commuting matrices $\mathcal{M}_{1}, \mathcal{M}_{2}$, selected from those obtained by Algorithm 1. In particular, it can be applied if conditions of Theorem 7 hold.

Algorithm 2 (The construction of a solution of the moment problem).

Input: commuting matrices $\mathcal{M}_{1}, \mathcal{M}_{2}$, selected from those obtained by Algorithm 1.

Step 1. Find all eigenvalues and eigenvectors of matrices $\mathcal{M}_{1}, \mathcal{M}_{2}$.

Step 2. Calculate the (atomic) solution of the moment problem by formula (24). Observe that the solution can have atoms at points $(x, y)$, where $x$ is an eigenvalue of $\mathcal{M}_{1}, y$ is an eigenvalue of $\mathcal{M}_{2}$. Notice that

$$
\mu(\{(x, y)\})=\left(E(\{(x, y)\}) g_{0}, g_{0}\right)_{H}=\left(E_{2}(\{y\}) g_{0}, E_{1}(\{x\}) g_{0}\right)_{H},
$$

where $E_{j}(\delta)(\delta \in \mathfrak{B}(\mathbb{R}))$ is the spectral measure of the (bounded) self-adjoint operator $\widetilde{M}_{j}, j=1,2$.

Output: $a$ solution $\mu$ of the moment problem.

Let us illustrate the above algorithms by the following examples.

Example 3. Consider the truncated moment problem (1) with $n=2, K=K_{1,1}$ (see Example 1), $\mathcal{K}=K+K=K_{2,2}$, and the following moments:

$$
\begin{aligned}
& s_{(0,0)}=4, s_{(0,1)}=12, s_{(0,2)}=48, \\
& s_{(1,0)}=4, s_{(1,1)}=12, s_{(1,2)}=48,
\end{aligned}
$$




$$
s_{(2,0)}=4, s_{(2,1)}=12, s_{(2,2)}=48 .
$$

Order the elements of $K$ as follows:

$$
\mathbf{k}_{0}=(0,0), \mathbf{k}_{1}=(0,1), \mathbf{k}_{2}=(1,0), \mathbf{k}_{3}=(1,1) .
$$

Thus, we have $\rho=3$.

The matrix $\Gamma=\left(s_{\mathbf{k}_{j}+\mathbf{k}_{m}}\right)_{m, j=0}^{3}$ has the following form:

$$
\Gamma=\left(\begin{array}{cccc}
4 & 12 & 4 & 12 \\
12 & 48 & 12 & 48 \\
4 & 12 & 4 & 12 \\
12 & 48 & 12 & 48
\end{array}\right)
$$

The non-negativity of $\Gamma$ holds. It is verified by checking that the determinants of all submatrices, standing on the intersections of rows and columns with the same indices, are non-negative. Observe that

$$
\Omega_{1}=\{0,1\}, \Omega_{2}=\{0,2\} .
$$

The matrices $\Gamma_{1}, \Gamma_{2}, \widehat{\Gamma}_{1}, \widehat{\Gamma}_{2}$ have the following forms:

$$
\begin{array}{cc}
\Gamma_{1}=\left(\begin{array}{cc}
4 & 12 \\
12 & 48
\end{array}\right), \quad \Gamma_{2}=\left(\begin{array}{ll}
4 & 4 \\
4 & 4
\end{array}\right), \\
\widehat{\Gamma}_{1}=\left(\begin{array}{cc}
4 & 12 \\
12 & 48
\end{array}\right), \quad \widehat{\Gamma}_{2}=\left(\begin{array}{ll}
48 & 48 \\
48 & 48
\end{array}\right) .
\end{array}
$$

Therefore conditions (17) hold. Let us apply Algorithm 1.

Step 1. Consider the associated Hilbert space H. Consider the multiplication operators $M_{l}$ as in (18). Notice that

$$
\begin{aligned}
& M_{1} g_{0}=g_{2}, \quad M_{1} g_{1}=g_{3}, \\
& M_{2} g_{0}=g_{1}, \quad M_{2} g_{2}=g_{3},
\end{aligned}
$$

and

$$
D\left(M_{1}\right)=\operatorname{Lin}\left\{g_{0}, g_{1}\right\}, \quad D\left(M_{2}\right)=\operatorname{Lin}\left\{g_{0}, g_{2}\right\} .
$$

Step 2. Let us apply the Gram-Schmidt orthogonalization process, removing linearly dependent elements, to the sequence $g_{0}, g_{1}, g_{2}, g_{3}$. We shall use the property (26). We obtain that

$$
f_{0}=\frac{1}{2} g_{0}, \quad f_{1}=\frac{1}{2 \sqrt{3}}\left(g_{1}-3 g_{0}\right),
$$

and

$$
g_{2}=g_{0}, \quad g_{3}=g_{1} \text {. }
$$

Therefore $\mathfrak{F}:=\left\{f_{0}, f_{1}\right\}$ is an orthonormal basis in $H$, and $\rho^{\prime}=1$.

Step 3. Notice that

$$
\Omega_{1}^{\prime}=\{2,3\}, \Omega_{2}^{\prime}=\{1,3\} .
$$

By (66) and (68) we see that $D\left(M_{1}\right)=H$ and $D\left(M_{2}\right)=\operatorname{Lin}\left\{g_{0}\right\}$. Therefore $\widetilde{M}_{1}=M_{1}$. Define $\widetilde{M}_{2}$ on $g_{1}$ in the following way:

$$
\widetilde{M}_{2} g_{1}=\sum_{j=0}^{1}\left(\alpha_{2 ; 1, j}+\beta_{2 ; 1, j} i\right) f_{j}, \quad \alpha_{2 ; 1, j}, \beta_{2 ; 1, j} \in \mathbb{R} .
$$

Since $g_{3}=g_{1}$, the procedure of the extension is finished. 
Step 4. By (64),(68) we see that $M_{1}=\widetilde{M}_{1}=E_{H}$. Therefore, $\mathcal{M}_{1}$ is the identity matrix. Let us calculate $\mathcal{M}_{2}$. Observe that

$$
g_{0}=2 f_{0}, \quad g_{1}=6 f_{0}+2 \sqrt{3} f_{1} .
$$

By (67),(71),(65),(70) we get

$$
\begin{gathered}
\widetilde{M}_{2} f_{0}=3 f_{0}+\sqrt{3} f_{1}, \\
\widetilde{M}_{2} f_{1}=\left(\frac{1}{2 \sqrt{3}}\left(\alpha_{2 ; 1,0}+\beta_{2 ; 1,0} i\right)-18\right) f_{0}+\left(\frac{1}{2 \sqrt{3}}\left(\alpha_{2 ; 1,1}+\beta_{2 ; 1,1} i\right)-6 \sqrt{3}\right) f_{1} .
\end{gathered}
$$

Then

$$
\mathcal{M}_{2}=\left(\begin{array}{cc}
3 & \frac{1}{2 \sqrt{3}}\left(\alpha_{2 ; 1,0}+\beta_{2 ; 1,0} i\right)-18 \\
\sqrt{3} & \frac{1}{2 \sqrt{3}}\left(\alpha_{2 ; 1,1}+\beta_{2 ; 1,1} i\right)-6 \sqrt{3}
\end{array}\right) .
$$

Step 5. Conditions (57) imply that

$$
\alpha_{2 ; 1,0}=36 \sqrt{3}+6, \quad \beta_{2 ; 1,0}=\beta_{2 ; 1,0}=0 .
$$

Conditions (57) are satisfied, since $\mathcal{M}_{1}$ is the identity matrix. The real parameter $\alpha_{2 ; 1,1}$ is free. Algorithm 1 is finished.

We see that $M_{1}$ is self-adjoint, therefore there is no need to verify condition (28) of Theorem 2. We choose $\alpha_{2 ; 1,1}=36+2 \sqrt{3}$ to get

$$
\mathcal{M}_{2}=\left(\begin{array}{cc}
3 & \sqrt{3} \\
\sqrt{3} & 1
\end{array}\right) .
$$

Matrices $\mathcal{M}_{1}, \mathcal{M}_{2}$ commute. We may apply Algorithm 2.

Step 1. The matrix $\mathcal{M}_{1}$ has an eigenvalue $\lambda_{1}=1$ and the eigensubspace $\mathcal{H}_{1}=H$. The matrix $\mathcal{M}_{2}$ has eigenvalues $\widetilde{\lambda}_{1}=0$ and $\widetilde{\lambda}_{2}=4$, with eigensubspaces

$$
\widetilde{\mathcal{H}}_{1}=\operatorname{Lin}\left\{-\frac{1}{2} f_{0}+\frac{\sqrt{3}}{2} f_{1}\right\}, \quad \widetilde{\mathcal{H}}_{2}=\operatorname{Lin}\left\{\frac{\sqrt{3}}{2} f_{0}+\frac{1}{2} f_{1}\right\},
$$

respectively.

Step 2. Observe that

$$
\begin{aligned}
& E_{2}(\{0\}) g_{0}=P_{\widetilde{\mathcal{H}}_{1}} g_{0}=\frac{1}{2} f_{0}-\frac{\sqrt{3}}{2} f_{1}, \\
& E_{2}(\{4\}) g_{0}=P_{\widetilde{\mathcal{H}}_{2}} g_{0}=\frac{3}{2} f_{0}+\frac{\sqrt{3}}{2} f_{1} .
\end{aligned}
$$

By formula (59) we obtain that the solution $\mu$ is 2-atomic with jumps 1, 3 at points $(1,0)$ and $(1,4)$, respectively.

In the case where operators $M_{1}, M_{2}$ are both nonself-adjoint, the commutativity condition (58) should be analyzed to get a solution of the moment problem. The following example illustrate this situation.

Example 4. Consider the truncated moment problem (1) with $n=2, K=K_{1,1}$ (see Example 1), $\mathcal{K}=K+K=K_{2,2}$, and the following moments:

$$
\begin{aligned}
& s_{(0,0)}=3, s_{(0,1)}=2, s_{(0,2)}=2, \\
& s_{(1,0)}=3, s_{(1,1)}=2, s_{(1,2)}=2, \\
& s_{(2,0)}=5, s_{(2,1)}=4, s_{(2,2)}=4 .
\end{aligned}
$$

The matrix $\Gamma=\left(s_{\mathbf{k}_{j}+\mathbf{k}_{m}}\right)_{m, j=0}^{3}$ has the following form:

$$
\Gamma=\left(\begin{array}{llll}
3 & 2 & 3 & 2 \\
2 & 2 & 2 & 2 \\
3 & 2 & 5 & 4 \\
2 & 2 & 4 & 4
\end{array}\right) .
$$


The non-negativity of $\Gamma$ holds. Observe that

$$
\Omega_{1}=\{0,1\}, \Omega_{2}=\{0,2\} .
$$

The matrices $\Gamma_{1}, \Gamma_{2}, \widehat{\Gamma}_{1}, \widehat{\Gamma}_{2}$ have the following forms:

$$
\begin{array}{ll}
\Gamma_{1}=\left(\begin{array}{ll}
3 & 2 \\
2 & 2
\end{array}\right), & \Gamma_{2}=\left(\begin{array}{ll}
3 & 3 \\
3 & 5
\end{array}\right), \\
\widehat{\Gamma}_{1}=\left(\begin{array}{ll}
5 & 4 \\
4 & 4
\end{array}\right), & \widehat{\Gamma}_{2}=\left(\begin{array}{ll}
2 & 2 \\
2 & 4
\end{array}\right) .
\end{array}
$$

Therefore conditions (17) hold. We can apply Algorithm 1.

Step 1. The same as in the previous example.

Step 2. Let us apply the Gram-Schmidt orthogonalization process, removing linearly dependent elements, to the sequence $g_{0}, g_{1}, g_{2}, g_{3}$. We shall use the property (26). We obtain that

$$
f_{0}=\frac{1}{\sqrt{3}} g_{0}, \quad f_{1}=\sqrt{\frac{3}{2}}\left(g_{1}-\frac{2}{3} g_{0}\right), \quad f_{2}=\frac{1}{\sqrt{2}}\left(g_{2}-g_{0}\right),
$$

and

$$
g_{3}=-g_{0}+g_{1}+g_{2} .
$$

Therefore $\mathfrak{F}:=\left\{f_{0}, f_{1}, f_{2}\right\}$ is an orthonormal basis in $H$, and $\rho^{\prime}=2$.

Step 3. Notice that

$$
\Omega_{1}^{\prime}=\{2,3\}, \Omega_{2}^{\prime}=\{1,3\} .
$$

Define $\widetilde{M}_{1}$ on $g_{2}$ in the following way:

$$
\widetilde{M}_{1} g_{2}=\sum_{j=0}^{2}\left(\alpha_{1 ; 2, j}+\beta_{1 ; 2, j} i\right) f_{j}, \quad \alpha_{1 ; 2, j}, \beta_{1 ; 2, j} \in \mathbb{R} .
$$

We define $\widetilde{M}_{2}$ on $g_{1}$ by the following formula:

$$
\widetilde{M}_{2} g_{1}=\sum_{j=0}^{2}\left(\alpha_{2 ; 1, j}+\beta_{2 ; 1, j} i\right) f_{j}, \quad \alpha_{2 ; 1, j}, \beta_{2 ; 1, j} \in \mathbb{R} .
$$

Since $g_{3}=-g_{0}+g_{1}+g_{2}$, the procedure of the extension is finished. Notice that we have 12 free real parameters at this moment.

Step 4. Observe that

$$
\begin{gathered}
g_{0}=\sqrt{3} f_{0}, \quad g_{1}=\frac{2}{\sqrt{3}} f_{0}+\sqrt{\frac{2}{3}} f_{1}, \quad g_{2}=\sqrt{3} f_{0}+\sqrt{2} f_{2}, \\
g_{3}=\frac{2}{\sqrt{3}} f_{0}+\sqrt{\frac{2}{3}} f_{1}+\sqrt{2} f_{2} .
\end{gathered}
$$

By (79),(84),(82),(83),(64) and (65) we get

$$
\begin{gathered}
\widetilde{M}_{1} f_{0}=f_{0}+\sqrt{\frac{2}{3}} f_{2}, \quad \widetilde{M}_{1} f_{1}=f_{1}+\frac{1}{\sqrt{3}} f_{2}, \\
\widetilde{M}_{1} f_{2}=\frac{1}{\sqrt{2}}\left(\alpha_{1 ; 2,0}+\beta_{1 ; 2,0} i-\sqrt{3}\right) f_{0}+\frac{1}{\sqrt{2}}\left(\alpha_{1 ; 2,1}+\beta_{1 ; 2,1} i\right) f_{1}+ \\
+\frac{1}{\sqrt{2}}\left(\alpha_{1 ; 2,2}+\beta_{1 ; 2,2} i-\sqrt{2}\right) f_{2} \\
\widetilde{M}_{2} f_{0}=\frac{2}{3} f_{0}+\frac{\sqrt{2}}{3} f_{1},
\end{gathered}
$$




$$
\begin{gathered}
\widetilde{M}_{2} f_{1}=\left(\sqrt{\frac{3}{2}}\left(\alpha_{2 ; 1,0}+\beta_{2 ; 1,0} i\right)-\frac{2 \sqrt{2}}{3}\right) f_{0^{+}} \\
+\left(\sqrt{\frac{3}{2}}\left(\alpha_{2 ; 1,1}+\beta_{2 ; 1,1} i\right)-\frac{2}{3}\right) f_{1}+\sqrt{\frac{3}{2}}\left(\alpha_{2 ; 1,2}+\beta_{2 ; 1,2} i\right) f_{2}, \\
\widetilde{M}_{2} f_{2}=f_{2} .
\end{gathered}
$$

Then

$$
\begin{gathered}
\mathcal{M}_{1}=\left(\begin{array}{ccc}
1 & 0 & \frac{1}{\sqrt{2}}\left(\alpha_{1 ; 2,0}+\beta_{1 ; 2,0} i-\sqrt{3}\right) \\
0 & 1 & \frac{1}{\sqrt{2}}\left(\alpha_{1 ; 2,1}+\beta_{1 ; 2,1} i\right) \\
\sqrt{\frac{2}{3}} & \frac{1}{\sqrt{3}} & \frac{1}{\sqrt{2}}\left(\alpha_{1 ; 2,2}+\beta_{1 ; 2,2} i-\sqrt{2}\right)
\end{array}\right), \\
\mathcal{M}_{2}=\left(\begin{array}{ccc}
\frac{2}{3} & \sqrt{\frac{3}{2}}\left(\alpha_{2 ; 1,0}+\beta_{2 ; 1,0} i\right)-\frac{2 \sqrt{2}}{3} & 0 \\
\frac{\sqrt{2}}{3} & \sqrt{\frac{3}{2}}\left(\alpha_{2 ; 1,1}+\beta_{2 ; 1,1} i\right)-\frac{2}{3} & 0 \\
0 & \sqrt{\frac{3}{2}}\left(\alpha_{2 ; 1,2}+\beta_{2 ; 1,2} i\right) & 1
\end{array}\right) .
\end{gathered}
$$

Step 5. Conditions (57) imply that

$$
\begin{gathered}
\beta_{1 ; 2, j}=\beta_{2 ; 1, j}=0, \quad j=0,1,2 ; \\
\alpha_{1 ; 2,0}=\frac{5}{\sqrt{3}}, \alpha_{1 ; 2,1}=\sqrt{\frac{2}{3}}, \alpha_{2 ; 1,0}=\frac{2}{\sqrt{3}}, \alpha_{2 ; 1,2}=0 .
\end{gathered}
$$

Two free real parameters remain: $\alpha_{1 ; 2,2}$ and $\alpha_{2 ; 1,1}$. Matrices $\mathcal{M}_{1}, \mathcal{M}_{2}$ take the following form:

$$
\begin{aligned}
& \mathcal{M}_{1}=\left(\begin{array}{cccc}
1 & 0 & \sqrt{\frac{2}{3}} \\
0 & 1 & \frac{1}{\sqrt{3}} \\
\sqrt{\frac{2}{3}} & \frac{1}{\sqrt{3}} & \frac{1}{\sqrt{2}} \alpha_{1 ; 2,2}-1
\end{array}\right), \\
& \mathcal{M}_{2}=\left(\begin{array}{cccc}
\frac{2}{3} & \begin{array}{c}
\frac{\sqrt{2}}{3} \\
\frac{\sqrt{2}}{3}
\end{array} & \sqrt{\frac{3}{2}} \alpha_{2 ; 1,1}-\frac{2}{3} & 0 \\
0 & 0 & 1
\end{array}\right) .
\end{aligned}
$$

Algorithm 1 is finished.

Condition (58) will be satisfied if $\alpha_{2 ; 1,1}=\sqrt{\frac{2}{3}}$. One free real parameter remains $\alpha_{1 ; 2,2}$. We set $\alpha_{1 ; 2,2}=2 \sqrt{2}$. Therefore

$$
\mathcal{M}_{1}=\left(\begin{array}{ccc}
1 & 0 & \sqrt{\frac{2}{3}} \\
0 & 1 & \frac{1}{\sqrt{3}} \\
\sqrt{\frac{2}{3}} & \frac{1}{\sqrt{3}} & 1
\end{array}\right), \quad \mathcal{M}_{2}=\left(\begin{array}{ccc}
\frac{2}{3} & \frac{\sqrt{2}}{3} & 0 \\
\frac{\sqrt{2}}{3} & \frac{1}{3} & 0 \\
0 & 0 & 1
\end{array}\right) \text {. }
$$

We may apply Algorithm 2.

Step 1. The matrix $\mathcal{M}_{1}$ has eigenvalues $\lambda_{0}=0, \lambda_{1}=1$ and $\lambda_{2}=2$, with eigensubspaces

$$
\begin{gathered}
\mathcal{H}_{0}=\operatorname{Lin}\left\{-\frac{1}{\sqrt{3}} f_{0}-\frac{1}{\sqrt{6}} f_{1}+\frac{1}{\sqrt{2}} f_{2}\right\}, \quad \mathcal{H}_{1}=\operatorname{Lin}\left\{\frac{1}{\sqrt{3}} f_{0}-\sqrt{\frac{2}{3}} f_{1}\right\}, \\
\mathcal{H}_{2}=\operatorname{Lin}\left\{\frac{1}{\sqrt{3}} f_{0}+\frac{1}{\sqrt{6}} f_{1}+\frac{1}{\sqrt{2}} f_{2}\right\},
\end{gathered}
$$

respectively. The matrix $\mathcal{M}_{2}$ has eigenvalues $\widetilde{\lambda}_{0}=0$ and $\widetilde{\lambda}_{1}=1$, with eigensubspaces

$$
\widetilde{\mathcal{H}}_{0}=\operatorname{Lin}\left\{-\frac{1}{\sqrt{3}} f_{0}+\sqrt{\frac{2}{3}} f_{1}\right\},
$$




$$
\widetilde{\mathcal{H}}_{1}=\operatorname{Lin}\left\{\frac{1}{\sqrt{2}} f_{0}+\frac{1}{2} f_{1}+\frac{1}{2} f_{2} ;-\frac{1}{\sqrt{6}} f_{0}-\frac{1}{2 \sqrt{3}} f_{1}+\frac{\sqrt{3}}{2} f_{2}\right\},
$$

respectively.

Step 2. Observe that

$$
\begin{gathered}
E_{1}(\{0\}) g_{0}=P_{\mathcal{H}_{0}} g_{0}=\frac{1}{\sqrt{3}} f_{0}-\frac{1}{\sqrt{6}} f_{1}-\frac{1}{\sqrt{2}} f_{2}, \\
E_{1}(\{1\}) g_{0}=P_{\mathcal{H}_{1}} g_{0}=\frac{1}{\sqrt{3}} f_{0}-\sqrt{\frac{2}{3}} f_{1}, \\
E_{1}(\{2\}) g_{0}=P_{\mathcal{H}_{2}} g_{0}=\frac{1}{\sqrt{3}} f_{0}+\frac{1}{\sqrt{6}} f_{1}+\frac{1}{\sqrt{2}} f_{2} ; \\
E_{2}(\{0\}) g_{0}=P_{\widetilde{\mathcal{H}}_{0}} g_{0}=\frac{1}{\sqrt{3}} f_{0}-\sqrt{\frac{2}{3}} f_{1}, \\
E_{2}(\{1\}) g_{0}=P_{\widetilde{\mathcal{H}}_{1}} g_{0}=\frac{2 \sqrt{3}}{3} f_{0}+\frac{\sqrt{6}}{3} f_{1} .
\end{gathered}
$$

By formula (59) we obtain that the solution $\mu$ is 3-atomic with unit jumps at points $(0,1),(1,0)$ and $(2,1)$.

Remark 2. Observe that in Algorithm 1 we restricted ourselves by considering possible extensions $\widetilde{M}_{j}$ of $M_{j}$ inside the original Hilbert space $H$. Instead of $H$ one can consider any finite-dimensional Hilbert space $\widetilde{H} \supseteq H$, and construct possible extensions $\widetilde{M}_{j}$ of $M_{j}$ in $\widetilde{H}$.

Acknowledgement: The author is grateful to Prof. Vasilescu for a useful discussion on the moment problems. The author is grateful to referees for their valuable comments and suggestions

\section{References}

[1] Yu. M. Berezansky, Expansions in Eigenfunctions of Selfadjoint Operators, (Amer. Math. Soc., Providence, RI, 1968)

[2] C. Berg, J. P. R. Christensen, P. Ressel, Harmonic Analysis on Semigroups, (Springer-Verlag, New York, 1984)

[3] D. Cichoń, J. Stochel, F. H. Szafraniec, Extending positive definiteness, Trans. Amer. Math. Soc., 363, No. 1 (2011), $545-577$.

[4] R. Curto, L. Fialkow, Solution of the Truncated Complex Moment Problem for Flat Data, (Memoirs Amer. Math. Soc. 119, no. 568 (1996), x+52 pp.)

[5] R. Curto, L. Fialkow, Flat Extensions of Positive Moment Matrices: Recursively Generated Relations, (Memoirs Amer. Math. Soc. 136, no. 648 (1998), x+56 pp.)

[6] L. A. Fialkow, Solution of the truncated moment problem with variety $y=x^{3}$, Trans. Amer. Math. Soc., 363, No. 6 (2011), 3133-3165.

[7] B. Fuglede, The multidimensional moment problem, Expo. Math., 1 no. 4 (1983), 47-65.

[8] D. P. Kimsey, M. Putinar, Complex orthogonal polynomials and numerical quadrature via hyponormality, Comput. Methods Funct. Theory, (2018), 1-16.

[9] D. P. Kimsey, H. J. Woerdeman, The truncated matrix-valued K-moment problem on $\mathbb{R}^{d}, \mathbb{C}^{d}$, and $\mathbb{T}^{d}$, Trans. Am. Math. Soc. 365, 10 (2013), 5393-5430.

[10] D. P. Kimsey, The subnormal completion problem in several variables, J. Math. Anal. Appl. 434, 2 (2016), $1504-1532$.

[11] J.-B. Lasserre, Moments, Positive Polynomials and Their Applications, (World Scientific, 2010)

[12] M. Laurent, B. Mourrain, A generalized flat extension theorem for moment matrices, Arch. Math. 93, (2009), 87-98.

[13] M. Marshall, Positive Polynomials and Sums of Squares, (Amer. Math. Soc., Math. Surveys and Monographs, Vol. 146, 2008)

[14] I. E. Ovcharenko, Two-dimensional power moment sequences, (in Russian) Ukrain. Mat. Zh. 36, no. 1 (1984), 51-56.

[15] I. E. Ovcharenko, Two-dimensional Hausdorff moment sequences, (in Russian) Ukrain. Mat. Zh. 36, no. 6 (1984), 729-733.

[16] M. Putinar, F.-H. Vasilescu, Solving moment problems by dimensional extension, Annals of Math., 149 (1999), $1087-1107$.

[17] K. Schmüdgen, The K-moment problem for compact semi-algebraic sets, Math. Ann., 289 (1991), 203-206.

[18] K. Schmüdgen, On the moment problem of closed semi-algebraic sets, J. reine angew. Math., 558 (2003), 225-234.

[19] Ph. J. di Dio, Konrad Schmüdgen, The multidimensional truncated moment problem: atoms, determinacy, and core variety, arXiv:1703.01497

[20] J. A. Shohat, J. D. Tamarkin, The Problem of Moments, (Amer. Math. Soc., 531 West 116th Street, New York City, 1943)

[21] F.-H. Vasilescu, Hamburger and Stieltjes moment problems in several variables, Trans. Amer. Math. Soc., 354 (2001), 12651278. 
[22] F.-H. Vasilescu, Dimensional stability in truncated moment problems, J. Math. Anal. Appl., 388 (2012), 219-230.

[23] F.-H. Vasilescu, An idempotent approach to truncated moment problems, Integr. Equ. Oper. Theory, 79 Issue 3 (2014), $301-$ 335.

[24] S. Yoo, Sextic moment problems on 3 parallel lines, Bull. Korean. Math. Soc., 54, No. 1 (2017), 299-318.

[25] S. M. Zagorodnyuk, On the two-dimensional moment problem, Ann. Funct. Anal. , 1, no. 1 (2010), 80-104.

[26] S.M. Zagorodnyuk, Generalized resolvents of symmetric and isometric operators: the Shtraus approach, Annals of Functional Analysis, 4, No. 1 (2013), 175-285.

[27] S. M. Zagorodnyuk, The two-dimensional moment problem in a strip, Methods Funct. Anal. Topology, 19, no. 1 (2013), $40-54$.

[28] S. M. Zagorodnyuk, The Nevanlinna-type parametrization for the operator Hamburger moment problem, J. Adv. Math. Stud., 10, No. 2 (2017), 183-199.

[29] S. Zagorodnyuk, On the truncated two-dimensional moment problem, Adv. Oper. Theory, 3, no. 2 (2018), 63-74. 